\title{
Cis-trans interactions of cell surface receptors: biological roles and structural basis
}

\author{
Werner Held · Roy A. Mariuzza
}

Received: 1 August 2011/Revised: 8 August 2011/Accepted: 8 August 2011 / Published online: 24 August 2011

(C) Springer Basel AG 2011

\begin{abstract}
Cell surface receptors bind ligands expressed on other cells (in trans) in order to communicate with neighboring cells. However, an increasing number of cell surface receptors are found to also interact with ligands expressed on the same cell (in cis). These observations raise questions regarding the biological role of such cis interactions. Specifically, it is important to know whether cis and trans binding have distinct functional effects and, if so, how a single cell discriminates between interactions in cis versus trans. Further, what are the structural features that allow certain cell surface receptors to engage ligand both on the same as well as on an apposed cell membrane? Here, we summarize known examples of receptors that display cis-trans binding and discuss the emerging diversity of biological roles played by these unconventional two-way interactions, along with their structural basis.
\end{abstract}

Keywords NK cell receptor - Ly49 - LILRB/PIR-B · MHC · Notch · Cis interaction - Trans interaction · NK cell education

W. Held $(\bowtie)$

Ludwig Center for Cancer Research of the University of Lausanne, 1066 Epalinges, Switzerland

e-mail: Werner.Held@unil.ch

\section{R. A. Mariuzza}

University of Maryland Biotechnology Institute for Bioscience and Biotechnology Research, Rockville, MD 20850, USA

R. A. Mariuzza

Department of Cell Biology and Molecular Genetics, University of Maryland, College Park, MD 20742, USA

\author{
Abbreviations \\ BCR B cell receptor \\ BTLA B and T lymphocyte attenuator \\ HVEM Herpesvirus entry mediator \\ Ig Immunoglobulin \\ LILRB Leukocyte immunoglobulin-like receptor B \\ MHC-I Major histocompatibility complex class I \\ PIR-B Paired immunoglobulin-like receptor-B \\ Siglec Sialic acid-binding immunoglobulin-like lectin
}

\section{Introduction}

One of the first cell surface receptors shown to bind ligand present in the plane of the same cell membrane was CD22, a sialic acid-binding immunoglobulin-like lectin (Siglec) expressed by B cells. Most CD22 was found to be "masked" by binding sialic acids attached to galactose (Sia $\alpha 2-6 \mathrm{Gal}$ ) on N-linked glycans of glycoproteins expressed on the B cell itself [1]. Sialidase treatment of B cells was sufficient to unmask CD22 and allow binding of exogenous sialoside probes. The preferential binding in cis is explained by the high concentration of sialic acid on the $\mathrm{B}$ cell surface $(25-30 \mathrm{mM})$, which is around 100 -fold higher than the $\mathrm{K}_{\mathrm{d}}$ of CD22 for sialic acid $(0.1-0.3 \mathrm{mM})$ [2]. Indeed, CD22 binding of higher-affinity multivalent sialoside probes does not require prior unmasking, and trans ligand can compete with cis ligand for CD22 binding in cell-cell conjugates [3]. These data suggest a dynamic equilibrium, in which CD22 can switch between cis and trans interactions, depending on the relative affinity/avidity of the respective ligands. However, the nature of the relevant sialic acid-modified cis ligand has remained elusive, although CD22 is associated with the B cell receptor 
(BCR), CD45 and CD22 itself [4]. Functional consequences of CD22 cis binding were proposed based on an analysis of human B cell lines. Separation of CD22 from the BCR in the B cell membrane by sequestration improved $B$ cell activation [5]. Similarly, B cells expressing a CD22 mutant that lacks sialic acid-binding function were hyperresponsive to stimulation through the BCR [6]. Cis binding is thus thought to set a signaling threshold, which prevents overstimulation of B cells after BCR cross-linking by antigen. This notion has been confirmed in several (but not all) mouse models, in which CD22 or sialic acid modifications have been disrupted completely or partially (for recent reviews, see [7, 8]). The work on Siglecs has provided a framework to investigate the role of cis-trans interactions in other receptor-ligand systems.

Renewed interest in the fact that certain cell surface receptors bind ligands expressed on the same membrane has stemmed from the analysis of natural killer (NK) cell receptors specific for major histocompatibility complex class I (MHC-I) molecules [9]. Such receptors were originally identified based on their ability to inhibit the effector function of $\mathrm{NK}$ cells in response to specific allelic variants of MHC-I molecules expressed on target cells [10, 11]. Some of these NK receptors were subsequently found to constitutively bind to the same MHC-I ligand expressed by the NK cell itself. This represented one of the first instances where cis-trans binding of a cell surface receptor was based on protein-protein interactions and where the cis and trans ligands were identical (for a review, see [12]).

Additional examples of receptors that can bind ligand in both cis and trans have since been reported [13-17], suggesting that such two-way interactions are more common than initially thought. In addition, it has emerged that cis interactions can serve distinct purposes and that there may be multiple structural solutions to the problem of how a cell surface receptor can engage the identical ligand on the same as well as on an apposed cell. Here, we summarize recent examples of receptor-ligand systems where evidence for cis and trans binding has been obtained. We also discuss the structural basis for this unconventional type of interaction and compare the biological roles of $c i$ s binding in these different systems. We reserve the term "cis interaction" for receptors that can bind the identical ligand expressed on the same and on an apposed cell, as schematically shown in Fig. 1.

\section{Biological roles of cis-trans interactions of cell surface receptors}

Cis association by inhibitory NK cell receptors specific for MHC-I

One of the first cell surface receptors shown to bind ligand in both cis and trans was the C-type lectin-like receptor Ly49A [9], an inhibitory receptor specific for multiple H-2D alleles expressed by NK cells [10]. Ly49A is the founding member of a small receptor family, which allows mouse NK cells to detect diseased host cells with nonphysiologically low levels of MHC-I molecules. Recognition of such cells is based on a dual receptor system consisting of activating and MHC-I-specific inhibitory receptors. Inhibitory receptors interrupt NK cell activation signaling when a cell's MHC-I levels are normal. When MHC-I levels are low, inhibitory signaling does not occur, which allows NK cell activation signaling to induce target cell lysis. This recognition strategy is classically known as "missing-self recognition" [18].

In addition to binding $\mathrm{H}-2 \mathrm{D}^{\mathrm{d}}$ expressed by potential target cells (in trans), Ly49A is associated with $\mathrm{H}-2 \mathrm{D}^{\mathrm{d}}$ expressed on the NK cell itself (in cis) [9]. To inhibit NK cell-mediated lysis, Ly49A binds $\mathrm{H}-2 \mathrm{D}^{\mathrm{d}}$ on target cells a

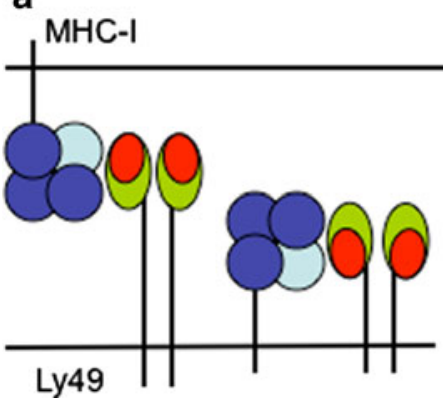

b

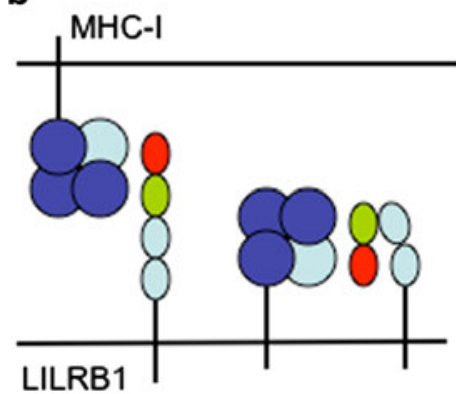

C

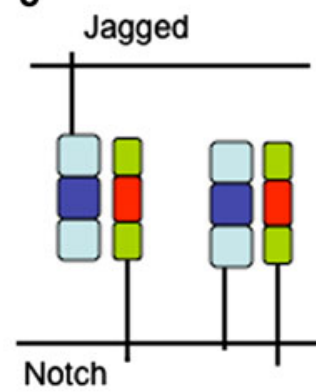

Fig. 1 Schematic representation of the structural basis for cis-trans interaction by cell surface receptors. a Ly49 receptors reverse the orientation of their ligand-binding domains relative to the NK cell membrane using a long flexible stalk region. b LILRB1 has a shorter stalk but is composed of four Ig domains, whereby MHC-I binding is mediated by the two membrane-distal Ig domains. Cis binding, which is likely based on a reversal of the orientation of the two distal $\mathrm{Ig}$ domains, may be enabled by flexibility of the short hinge between Ig domains 2 and 3. c Notch and Delta are rigid EGF repeat-containing receptors. Symmetric binding sites allow anti-parallel (trans) and parallel (cis) binding without a need for reversal of the ligand-binding domains 
Table 1 Cell surface receptors that interact with ligand expressed in trans and in cis

\begin{tabular}{|c|c|c|c|c|c|c|c|}
\hline Receptor & Structure & Cell type & Species & Receptor function & Ligand & Role of cis binding & Ref. \\
\hline Ly49A, C, I & CTLL & NK & Mouse & Inh. & MHC-Ia & Comp. Inh. & [9] \\
\hline Ly49Q & & $\mathrm{pDC}$ & Mouse & Act. & & $?$ & [27], [29] \\
\hline LILRB2 & Ig & Mast, DC & Human & Inh & MHC-Ia/b & Inh & [14] \\
\hline LILRB1 & & $\mathrm{T}, \mathrm{B}, \mathrm{NK}$ & & & & & [28] \\
\hline PIR-B & $\operatorname{Ig}$ & Mast, DC & Mouse & Inh & MHC-Ia/b & Inh & [14], [35] \\
\hline \multirow[t]{2}{*}{ HVEM } & TNFR & $\mathrm{T}$ & Human & Act & BTLA & Comp. Inh. & [16] \\
\hline & & & & & HSV env & Act & [16] \\
\hline EphA4 & & Neuron & Mouse & Act & Ephrin-A2 & Comp. Inh. & [13] \\
\hline Plexin-A4 & GAP, Ig & Neuron & Mouse & Act & Sema6a & Comp. Inh. & [17] \\
\hline \multirow[t]{2}{*}{ Notch1 } & EGF & & Human & Act & Delta-like1 & Comp. Inh. & {$[47]$} \\
\hline & & & & & Jagged1 & & [15] \\
\hline
\end{tabular}

CTLL C-type lectin like, $I g$ immunoglobulin-like, TNFR tumor necrosis factor related, EGF epidermal growth factor related, $G A P$ GTPase activating protein domain, $N K$ natural killer cells, $p D C$ plasmacytoid dendritic cells, $D C$ dendritic cell, Inh inhibitory, Act activating, Comp. inh . competitive inhibition of trans binding, $M H C$-Ia classical major histocompatibility complex class I molecules, $M H C$ - $I b$ non-classical MHC-I molecules, BTLA B- and T-lymphocyte attenuator, HSV env herpes simplex virus envelope

using a binding site beneath the peptide-binding platform of $H-2 D^{d}$ [19-21]. This same binding site allows the association of Ly49A with $\mathrm{H}-2 \mathrm{D}^{\mathrm{d}}$ in cis [9]. Indeed, the majority $(>70 \%)$ of Ly49A molecules is bound in cis [22, 23 ] and only a minority remains available to bind ligand in trans. Besides a high affinity for $\mathrm{H}-2 \mathrm{D}^{\mathrm{d}}$, Ly49A binds other $\mathrm{H}-2 \mathrm{D}$ alleles with variable affinities [24]. Interestingly, the affinity of Ly49A for a specific H-2D allele directly correlates with the number of receptors bound in cis. Consequently, the number of free receptors available to bind MHC-I in trans decreases when the affinity of Ly49A for ligand increases [25]. The association of Ly49A with $\mathrm{H}-2 \mathrm{D}^{\mathrm{d}}$ in cis is stable and, unlike Siglecs, there is no evidence that cis-bound Ly49A can switch to trans ligand during the formation of an immune synapse [22]. Finally, it is important to point out that NK cells expressing a cisengaged Ly49 receptor are fully functional, which implies that $c$ is association does not result in constitutive inhibitory signaling that renders $\mathrm{NK}$ cells non-functional. Collectively, therefore, cis association of Ly49A with MHC-I stably reduces the number of receptors that are available for functional interaction with MHC-I on target cells [22]. The fact that the number of available receptors is reduced when the affinity for H-2D is increased raises the possibility that $\mathrm{NK}$ cell inhibition via Ly49A is relatively independent of whether a given mouse strain expresses a high or a low affinity $\mathrm{H}-2 \mathrm{D}$ allele. Notwithstanding, in a given mouse strain, cis association lowers the threshold at which NK cell activation exceeds inhibition during target cell encounter [9]. Since cis association is stable, the function of NK cells is constitutively improved. This does not exclude the possibility that changes in the expression levels of MHC-I or Ly49 on the surface of NK cells could further modify the responsiveness of these effector cells.

Like Ly49A, most other inhibitory Ly49 receptors specific for MHC-I have the capacity to interact with MHC-I molecules in cis [26, 27] (Table 1). Besides Ly49 family receptors, $\mathrm{NK}$ cells use additional inhibitory receptors specific for MHC-I. These include heterodimeric C-type lectin-like CD94/NKG2 receptors, which are used by human and rodent NK cells, as well as structurally unrelated killer immunoglobulin-like receptors (KIRs) and leucocyte immunoglobulin-like receptor B1 (LILRB1) (also termed LIR-1 or ILT2) expressed by human NK cells. In contrast to Ly49 receptors, there is no evidence for cis binding in the case of the mouse CD94/NKG2A receptor or the human KIR family receptor KIR2DL1 (unpublished data). However, cis association has been reported for LILRB1 [28], which will be discussed below.

Certain receptors specific for MHC-I are not actually expressed by NK cells and some activate rather than inhibit cellular functions. For example, the MHC-I-specific Ly49Q receptor improves the response of plasmacytoid dendritic cells (pDC) to microbial infection. PDCs lacking Ly49Q produce low amounts of type I interferon in response to Toll-like receptor 9 (TLR9) stimulation by unmethylated microbial cytosine guanine dinucleotide (CpG) DNA [29]. Similarly, the response is also low in mice devoid of classical MHC-I molecules [29], suggesting that Ly49Q improves the response of TLR9 in connection with MHC-I binding. Indeed, Ly49Q co-localizes with MHC-I at the cell surface [30] and most, but not all, Ly49Q is physically associated with MHC-I in cis [27]. However, it is not formally known whether cis binding of MHC-I by Ly49Q 
plays an essential role. Moreover, it is not clear how Ly49Q improves the function of endosome-resident TLR9. One way in which TLR9 function can be improved was discovered based on an analysis of the human inhibitory NK cell receptor KIR3DL2 (p140), which was known to be specific for a small group of HLA-A alleles. This cell surface receptor directly binds CpG DNA and delivers it to endosomes, which improves cytokine production by NK cells in response to microbial DNA [31]. Therefore, in addition to MHC-I binding, members of structurally distinct human and mouse receptor families are also involved in TLR signaling. It will be interesting to see whether recognition of MHC-I and of CpG DNA are separate functions, or whether MHC-I and $\mathrm{CpG}$ recognition is instead coordinated. If so, the physiological function of MHC-I recognition in this innate immune response to microbes remains to be defined.

Subsequent to C-type lectin-like Ly49 receptors and KIR, immunoglobulin-like human LILRB1 and LILRB2 receptors and the homologous mouse PIR-B receptor were identified as a third type of inhibitory MHC-I receptor that is relatively broadly expressed by lymphoid, as well as myeloid, cells. Further, these receptors bind a wide range of classical and non-classical MHC-I molecules with low affinity and with limited allele specificity (reviewed in [32]). LILRB1 (ILT2) inhibits NK cell effector function in response to target cells expressing MHC-I [33], and LILRB/PIR receptors generally dampen the stimulation of immune responses upon binding to MHC-I molecules on antigen-presenting cells [33]. Subsequently, LILRB/PIR-B receptors were found to associate with MHC-I molecules in cis $[14,28]$. This extended ligand binding in cis by C-type lectin-like receptors to structurally unrelated Ig-like receptors suggested that cis binding of MHC-I impacts numerous hematopoietic cell types and is not restricted to NK cells.

Similarly to Ly49, the association between MHC-I and LILRB/PIR-B receptors in cis is constitutive [14]. However, in contrast to Ly49, the ITIMs of LILRB/PIR-B receptors are constitutively phosphorylated and cis association results in constitutive inhibition signaling [14]. In mast cells, the suppressive effect increases the threshold for degranulation upon activation through the $\mathrm{Fc}$ receptor for IgE, FceRI [14]. The interaction of LILRB2/PIR-B with MHC-I in cis reduces activation signaling and maturation in additional cell types. The expression of a specific HLA-B allele (HLA-B35x), for which LILRB2 has increased affinity, is associated with impaired maturation and cytokine production by dendritic cells (DC). It has been proposed that recognition of the DC's own HLA-B35x by LILRB2 contributes to this impairment and that this correlates with accelerated disease in HIV patients who carry the HLA-B35x allele [34]. Further, the activation of T cells by DCs is reduced due to competition between the CD8 coreceptor (on the T cell) and PIR-B (on the DC) for binding to MHC-I on the DC [35]. Collectively, therefore, cis association of LILRB/PIR-B receptors with MHC-I mediates a suppressive effect, which is further increased upon engagement of MHC-I in trans. Even though it is not known whether cis association of LILRB/PIR-B receptors is stable, it results in an increased threshold for cellular activation, similar to the role of cis binding by CD22. For LILRB/PIR-B receptors, it is not clear how the cis complex couples to the various activation receptors. In contrast, cis binding by Ly49 receptors mainly serves as a competitive inhibitor for trans binding, which lowers the activation threshold in NK cells. Together, these findings suggest that cis binding of MHC-I molecules can play distinct biological roles.

\section{NK cell education}

In addition to dampening the effector function of NK cells during target cell recognition, inhibitory receptors specific for MHC-I impact NK cell function in another way. Engagement of MHC-I, possibly during development, improves the function of activating NK cell receptors [26, 36-39]. This effect is known as NK cell "education" [40]. The improved functionality of activation receptors may be essential for NK cell reactions to cells lacking MHC-I in the absence of acute inflammation. While it is clear that engagement of MHC-I receptors induces functional changes, it is currently not possible to identify educated NK cells based on phenotypic changes. In addition, the precise role of inhibitory receptors for NK cell education is debated. The "licensing" model implies that NK cell activation receptors are by default hypo-active. They are rendered responsive when NK cells express an inhibitory receptor specific for self-MHC-I [26, 38]. Conversely, the "disarming" model [41] implies that activating NK cell receptors are by default active. They are rendered hypoactive due to continuous stimulation. This is prevented by the acquisition of an inhibitory receptor, which is specific for self-MHC [37]. Irrespective of the precise mechanism involved, both models imply that NK cell education depends on an interaction of inhibitory receptors with MHC-I expressed on other cells. However, the fact that most inhibitory Ly49 NK cell receptors also bind MHC-I on the NK cell itself raised the issue of whether NK cell education depends on MHC-I recognition in trans or in cis. This question was addressed using a Ly49 receptor variant, which binds MHC-I in trans, but fails to bind in cis. This was achieved by replacing the predicted flexible stalk region of Ly49A with a rigid stalk from another C-type lectin-like receptor, CD72, which should prevent cis binding by directing the ligand-binding domains of Ly49A 
away from the NK cell membrane. Indeed, this receptor variant failed to bind MHC-I in cis while retaining the ability to inhibit NK cell effector function in response to target cells expressing $\mathrm{H}-2 \mathrm{D}^{\mathrm{d}}$. Despite the fact that this engineered receptor was a functional inhibitory NK cell receptor, it was unable to educate NK cells [42]. These data dissociate the classical inhibitory from the educating function of an MHC-I receptor. Such dissociation is not compatible with "disarming", as this model relies exclusively on the inhibitory function of MHC-I receptors. In addition, the data suggest that cis interaction is necessary for NK cell education. In agreement with this conclusion, an increasing affinity of wild type Ly49A for MHC-I enhances NK cell education, and, as discussed above, this correlates with the extent to which Ly49A is associated with MHC-I in cis [25].

A key question, then, is how cis interaction contributes to NK cell education. Once again, sequestration of Ly49 by MHC-I expression in cis seems to play a role. We noted that unengaged Ly 49 receptors (i.e.. Ly49 receptors, which have no MHC-I ligand in a particular mouse strain, such as Ly49A in $\mathrm{H}-2^{\mathrm{b}}$ mice), dampen NK cell activation. In this situation, unengaged Ly49 receptors may be in proximity to activation receptors, thereby reducing their responsiveness. As a precedent, the proximity of the coinhibitory CD22 receptor is sufficient to dampen BCR stimulation, despite the fact that $\mathrm{CD} 22$ is not engaged by a trans ligand. In agreement with this model, the suppressive effect of unengaged Ly49A requires a functional cytoplasmic inhibition motif [43]. Finally, unengaged Ly49A, as well as KIR [43], accumulate at NK cell synapses formed with target cells, which lack the corresponding MHC-I ligand [42]. The presence of MHC-I ligand in cis is believed to sequester Ly49A away from activation receptors, which reverses the suppressive effect of unengaged Ly49A and improves the function of NK cell activation receptors.

As discussed above, NK cell education by Ly49A is dependent on cis interaction with MHC-I. This raises the question of how receptors, which do not bind MHC-I in cis (such as KIR2DL1 or CD94/NKG2A), are able to educate NK cells. One structural difference between these receptors is that KIR2DL1 and CD94/NKG2A contain two immunoreceptor tyrosine-based inhibition motifs (ITIMs) in their cytoplasmic domains. By contrast, each subunit of the homodimeric Ly49 receptors has a single ITIM. Perhaps the tandem arrangement of ITIMs can lead to distinct types of signals based on ligand binding in trans. Consistent with such a possibility, KIR2DL1 can play a costimulatory or an inhibitory role in $\mathrm{CD}^{+}{ }^{+} \mathrm{T}$ cell hybridomas [44]. For Ly49 receptors, their sequestration by MHC-I expression in cis, rather than signaling induced by cis binding, appears to be essential for NK cell education.
Irrespective of the precise mechanism, cis association of ITIM-bearing Ly49 receptors improves the function of mouse NK cells in two separate ways: it improves the function of activating NK cell receptors and it reduces the inhibitory capacity of MHC-I receptors. Both effects contribute to lowering the threshold at which NK cell activation will exceed inhibition signals, rendering NK cells more reactive and potentially more useful.

\section{Cis binding by activating receptors}

Cis binding was initially observed for inhibitory Ly 49 and LILRB receptors, while an activating Ly 49 family receptor was not influenced by the presence of its ligand in cis [45], raising the question of whether cis interaction is restricted to cell surface receptors with inhibitory function. However, this is not the case, as evidence for cis-trans binding has now been reported for activating receptors that recognize non-MHC ligands. A recent example includes the TNF family receptor herpes virus entry mediator (HVEM), which is subject to regulation by its ligand, B and T lymphocyte attenuator (BTLA), present on the $T$ cell membrane. HVEM engagement of BTLA expressed on neighboring cells results in bi-directional signaling: the HVEM-expressing cell is strongly co-stimulated while the BTLA-expressing cell is co-inhibited. This raised the question of how naïve HVEM-expressing T cells are kept in check. Recent data show that HVEM is constitutively associated with BTLA in cis and acts as a competitive inhibitor, which prevents HVEM clustering by ligand engagement in trans [16]. Consequently, HVEM-BTLA cis complexes are thought to maintain $\mathrm{T}$ cells in a naïve state. Upon T cell activation, BTLA is downregulated, which renders HVEM competent for signaling. These findings suggest that cis association makes the activating HVEM receptor functionally unavailable, whereas trans binding results in signaling. Similar to Ly49, cis binding of HVEM serves as a competitive inhibitor for trans binding.

Activating receptors that can bind the equivalent ligand expressed in cis and trans are not restricted to the immune system. Ephrin receptors (Eph) are involved in the sorting of distinct cell types in various tissues, such as the gut, or in directing the growth of axons. Cell sorting occurs based on cellular repulsion, which is induced when Eph binds ephrin ligands on other cells. Recent data suggest that the activity of certain Eph is influenced by ephrin expression by the same cell [46]. For example, the EphA receptor binds ephrinA ligand expressed in cis via the Eph ligand-binding domain [13]. Similar to HVEM, cis interaction does not induce Eph tyrosine kinase activity; rather, it reduces the interaction with ephrin on other cells, which dampens the repulsive signaling by Eph [13]. In a functionally related system, plexin receptors on neurons bind to semaphorin 
ligands on other cells (trans interaction) and transduce signals for regulating neuronal axon guidance. However, expression of Plexin-A4 and Semaphorin6A on the same neuron was found to attenuate the response to semaphorin ligands in trans [17]. This effect is mediated by the formation of stable complexes between Plexin-A4 and Semaphorin6A in cis, which prevent ligand binding in trans. In this way, the Plexin-A4-Semaphorin6A cis interaction serves as an inhibitory mechanism to modulate axon guidance.

Finally, recent data suggest that the activity of the Notch receptor is influenced by cis binding to its Delta ligand [15]. Notch induces distinct cell fates in groups of initially identical cells, a process referred to as lateral signaling. Delta has two activities: it activates Notch in neighboring cells and it inhibits Notch expressed on the same cell. The negative effect is based on the interaction of the two proteins in cis, which prevents trans binding by the receptor. Functional data demonstrate that the response of Notch to Delta expressed in trans is graded (i.e., it is proportional to the amount of Delta present on a neighboring cell). Delta expression in cis serves as a competitive inhibitor for Notch activation by trans Delta. Mathematical modeling and functional data showed that this generates a very sharp threshold, above which Notch responds to trans Delta [47]. This Notch-Delta switch may explain how very small differences in the relative concentrations of Notch and Delta on the surfaces of two cells can be translated into binary cell fates.

Altogether, there is now ample evidence from multiple distinct receptor-ligand systems that the ability of a cell surface receptor to respond to ligand on a neighboring cell may be subject to control by expression of the same ligand on the same cell. In most of these cases, cis binding does not induce receptor signaling. Rather, by competitively inhibiting trans binding, cis interaction modifies the threshold at which the cell will be activated.

\section{Cis binding and viral infection}

HVEM was originally identified as the receptor for herpes simplex virus envelope glycoprotein D (HSV gD) [48]. Based on the formation of a cis complex between HVEM and BTLA, the viral envelope protein $\mathrm{gD}$ was also tested for association with HVEM in cis. This was indeed the case; however, the functional consequence of this interaction was different from the HVEM-BTLA cis association. Whereas the HVEM-BTLA complex is functionally inert, the HVEM-gD cis complex activates NF- $\kappa \mathrm{B}$ signaling [16]. NF- $\kappa \mathrm{B}$ activation induces the expression of pro-survival genes and may provide infected cells with a selective survival advantage early during HSV infection. These data suggest that certain viruses exploit the ability of cell surface receptors to bind ligand in cis in order to generate cell-autonomous signals that favor infected cells.

Perhaps along the same lines, in addition to binding HLA molecules, LILRB1 recognizes the HLA-like molecule UL18 encoded by human cytomegalovirus (HCMV) [49]. The fact that LILRB1 binds MHC-I in cis raises the issue of whether UL18 might also interact with LILRB1 on the surface of HCMV-infected cells and, if so, whether this has functional consequences similar to or distinct from MHC-I binding in cis.

\section{Structural basis for cis versus trans interaction}

From a structural perspective, it is not obvious how a cell surface receptor can bind a ligand in both cis and trans using the identical binding site. In addition, it is not known why cis and trans binding results in distinct functional outcomes. These issues have been addressed for the interaction of Ly49 receptors with MHC-I ligand [50]. These studies revealed that Ly49 receptors adopt two distinct conformations to mediate cis-trans recognition.

Ly49 receptors are homodimeric type II glycoproteins, with each chain composed of a ligand-binding C-type lectin-like domain, the so-called natural killer receptor domain (NKD), which is connected by an unusually long stalk of around 70 residues to the transmembrane and cytoplasmic domains [51, 52]. Based on the crystal structure of Ly49L, which was the first to provide structural information regarding the stalk region, we proposed that the NKDs must back-fold onto a specific segment of the stalk region, the so-called $\alpha_{\mathrm{S}} 3$ segment, to bind MHC-I in trans (Fig. 2a). In agreement with this model, replacement of the $\alpha_{S} 3$ segment greatly reduces the ability of the Ly $49 \mathrm{~A}$ receptor to functionally engage MHC-I on other cells [50], validating the proposal that Ly 49 trans binding is mediated by an unusual receptor conformation in which the NKDs are back-folded onto the stalk.

The crystal structure of Ly49L without the stalk region revealed distinct non-covalent associations of the two NKDs in the absence as compared to the presence of the stalk. These data, together with the earlier co-crystal structure of Ly49A bound to H-2 ${ }^{d}$ [19], raised the possibility that Ly49 receptors assume an extended conformation, in which the $\alpha_{\mathrm{S}} 3$ segments of the stalk do not contact the NKDs, to bind MHC-I in cis (Fig. 2b). Indeed, the $\alpha_{\mathrm{S}} 3$ segment, which is essential for functional trans binding, is not required for cis binding, providing evidence that the NKDs are disengaged from the stalk during cis interactions. In addition, progressive shortening of the stalk region impaired trans rather than cis binding. This is expected if cis binding is mediated by an extended receptor conformation. Based on the model (Fig. 2), it is easy to 


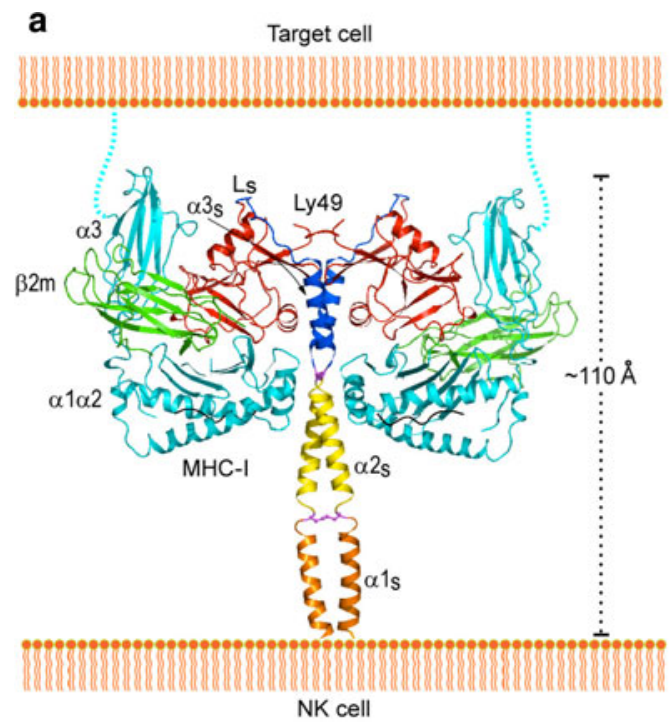

Fig. 2 Trans and cis interactions of Ly49 receptors with MHC-I ligands. a Trans interaction of an Ly49 receptor with two MHC-I molecules. The MHC-I heavy chain is cyan; $\beta_{2} \mathrm{~m}$ is green; the Ly49 NKD is red; the $\alpha 3_{\mathrm{S}}$ helix of the Ly49 stalk and loop $\mathrm{L}_{\mathrm{S}}$ connecting $\alpha 3_{\mathrm{S}}$ to the NKD are blue; the disulfide bond linking the $\alpha 3_{\mathrm{S}}$ helices is magenta. The predicted $\alpha 1_{\mathrm{S}}$ and $\alpha 2_{\mathrm{S}}$ helices of the stalk are drawn arbitrarily in orange and yellow, respectively, with the putative

envisage how cis binding by a receptor with a shortened stalk can be accommodated, while trans binding is impaired by structural clashes between the MHC-I molecules and the NK cell membrane. Finally, biochemical analyses confirmed that the cis complexes consist of an Ly49A homodimer associated with a single $\mathrm{D}^{\mathrm{d}}$ molecule, as predicted by the model (Fig. 2b). By contrast, trans complexes consist of an Ly49A homodimer associated with two $D^{\mathrm{d}}$ molecules (Fig. 2a) [50].

Cis and trans interactions of Ly49 NK cell receptors with MHC-I are thus mediated by two distinct receptor conformations. The two ligand-binding domains of Ly 49 are back-folded onto the long stalk region to bind two MHC-I molecules in trans. Dissociation of the ligandbinding domains from the stalk and their reorientation relative to the NK cell membrane allow monovalent binding of MHC-I in cis. The back-folded and extended configurations define the structural basis for cis-trans binding by Ly49 receptors (schematically shown in Fig. 1a).

Cis-trans binding may generally depend on large structural rearrangements that allow cell surface receptors to inverse the orientation of the ligand-binding domain relative to the cell membrane. While cis-trans binding of Ly49 receptors depends on an unusually long stalk ( $>70$ amino acids), the stalk regions of LILRB/PIR-B receptors are considerably shorter (42-52 amino acids). In addition, these receptors are composed of four or six extracellular Ig-like domains [33, 49], whereby the membrane distal D1 b

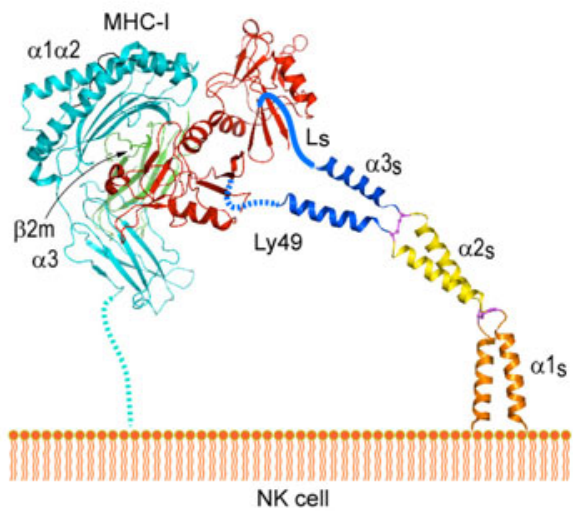

disulfide bond in magenta. The ligand-binding domains in the Ly49 homodimer are back-folded onto the stalk to bind two MHC-I molecules on a target cell. b Cis interaction of Ly49 with a single MHC-I molecule. The Ly49 receptor assumes an extended conformation, which engages a single MHC-I molecule on the NK cell itself. The ligand-binding domains are thereby dissociated from the stalk region. Reproduced with permission from Immunity [50]

and D2 domains mediate MHC-I binding. Based on the corresponding specificity of trans and cis binding [14], it seems likely that LILRB/PIR-B receptors use the same binding site for the two types of interactions. If that is indeed the case, the D1 and D2 domains of LILRB1 must reverse direction with respect to the effector cell surface to bind MHC-I in cis. This would require that the receptor bends back on itself to adopt a horseshoe-shaped configuration of the four Ig domains, implying considerable flexibility in the segment connecting the D2 and D3 domains. As a precedent, the four $\mathrm{N}$-terminal Ig-like domains of the Drosophila Dscam protein do assume a horseshoe arrangement, which depends on a very short, five-residue hinge between D2 and D3 [53]. In the case of PIB-B, which has six Ig-like domains, the segments connecting D4-D5 or D5-D6 may provide additional flexibility. Therefore, the ability to reverse the orientation of their ligand-binding domains may be a general feature of receptors that bind in cis and trans (schematically shown in Fig. 1b). Such reversals may be made possible by two distinct structural features: long stalk regions in the case of Ly49s and flexible interdomain hinges in the case of LILRB2 and PIR-B.

Recent data point to an additional structural solution for cis binding. As discussed above, Notch interacts with Delta in cis and trans. However, the extracellular domains of Notch and Delta are composed of EGF-like domains that are thought to form extended and rigid structures. Notwithstanding, structural and functional analyses suggest 
that Notch-ligand interactions use the same surfaces for $\mathrm{cis}$ and trans binding. In silico docking of crystal structures of the binding domains of Notch with that of its ligand, Jagged, provided evidence for two distinct receptor-ligand complexes [15]. An anti-parallel engagement of receptor and ligand is thought to mediate a trans interaction. Unexpectedly, there is also the possibility of a parallel engagement that would allow cis binding. Anti-parallel and parallel binding modes appear to be possible because the respective binding sites are symmetric. Even though this model remains to be further tested, symmetry of binding sites does represent, in principle, an additional solution to the structural problem of how cell surface receptors can bind the same ligand in cis and trans (schematically shown in Fig. 1c).

\section{Basis for the divergent outcomes of cis and trans binding}

An important unresolved issue is how cis and trans binding can result in distinct functional outcomes. Receptor engagement in trans is normally productive and results in receptor signaling. In contrast, binding of the same ligand in cis, using the same binding site, is often non-productive. The engagement of cell surface receptors induces intracellular signaling via two principal mechanisms: ligand-induced structural changes in the receptor and/or oligomerization of receptor-ligand complexes [54]. For Ly49 receptors, it was shown that Ly49C in complex with $\mathrm{H}-2 \mathrm{~K}^{\mathrm{b}}$ retains nearly the same structure as the free receptor [55], arguing against MHC-induced conformational changes in Ly49 as a signaling mechanism. On the other hand, monovalent human KIR-HLA-C complexes are thought to multimerize in a zinc-dependent fashion, and this seems to be needed for inhibitory signaling at the NK cell immune synapse [56]. Similarly, Ly49 signaling may depend on clustering of ligand-engaged Ly49 dimers. Although a single Ly49 receptor can associate with two MHC-I molecules to form a bivalent Ly49-MHC-I complex (Fig. 2a), it is not apparent how higher-order clustering of Ly49-MHC-I complexes might assemble. A prominent feature of the Ly49L structure, which contains a portion of the stalk region, is a flexible loop region that connects the $\alpha 3_{\mathrm{S}}$ segment with the NKD. This so-called $\mathrm{L}_{\mathrm{S}}$ loop is not needed for MHC-I binding in trans. Surprisingly, however, $\mathrm{L}_{\mathrm{S}}$ is essential for the inhibitory function of the Ly49A receptor and is required for receptor accumulation at the immune synapse. In the model of the bivalent Ly49-MHC-I trans complex (Fig. 2a), the $\mathrm{L}_{\mathrm{S}}$ loop is on the exterior of the complex, fully exposed to solvent. It is thus conceivable that $\mathrm{L}_{\mathrm{S}}$ mediates lateral interactions with other cell surface molecules or adjacent bivalent Ly49-MHC-I trans complexes, and that these lateral associations are required for receptor signaling. Importantly, in the cis complex, where the NKDs are dissociated from the stalk, the $\mathrm{L}_{\mathrm{s}}$ loop must adopt an extended conformation (Fig. 2b). This structural change may prevent $\mathrm{L}_{\mathrm{S}}$ from mediating lateral association of Ly49 receptors that are bound to MHC-I in cis. Accordingly, in situations where cis complexes are inert, cis engagement may be mediated by receptor conformations that cannot cluster.

\section{Concluding remarks}

A significant number of cell surface receptors have now been shown to be able to interact with ligands in both cis and trans. Two general scenarios emerge from these different receptor-ligand systems with regard to the role of $\mathrm{cis}$ interaction: First, the binding of a ligand to its receptor in cis is functionally inert and serves as a competitive inhibitor for receptor binding in trans. Second, the binding of a ligand to its receptor in cis results in tonic (inhibition) signaling, which is enhanced when the receptor is engaged in trans. In both cases, cis binding alters the threshold at which cells produce a response to an external stimulus. Cis binding may be stable to permanently modify the threshold for a response. Alternatively, cis and/or trans ligands may be subject to regulation or trans ligand may compete with cis ligand for receptor binding. In these cases, the threshold response may vary depending on the relative abundance of cis and trans ligand. Consequently, the various flavors of cis binding may allow for diverse ways in which receptor function is not only dependent on the presence of ligand on other cells but can also be modified by the presence of ligand on the same cell.

Acknowledgments W.H is supported in part by grants from the Swiss National Science Foundation and Oncosuissse. R.A.M. is supported by National Institutes of Health Grant AI47990.

\section{References}

1. Razi N, Varki A (1998) Masking and unmasking of the sialic acid-binding lectin activity of CD22 (Siglec-2) on B lymphocytes. Proc Natl Acad Sci USA 95:7469-7474

2. Collins BE, Blixt O, DeSieno AR, Bovin N, Marth JD, Paulson JC (2004) Masking of CD22 by cis ligands does not prevent redistribution of CD22 to sites of cell contact. Proc Natl Acad Sci USA 101:6104-6109

3. Collins BE, Blixt O, Han S, Duong B, Li H, Nathan JK, Bovin N, Paulson JC (2006) High-affinity ligand probes of CD22 overcome the threshold set by cis ligands to allow for binding, endocytosis, and killing of B cells. J Immunol 177:2994-3003

4. Han S, Collins BE, Bengtson P, Paulson JC (2005) Homomultimeric complexes of CD22 in B cells revealed by protein-glycan cross-linking. Nat Chem Biol 1:93-97

5. Doody GM, Justement LB, Delibrias CC, Matthews RJ, Lin J, Thomas ML, Fearon DT (1995) A role in B cell activation for 
CD22 and the protein tyrosine phosphatase SHP. Science 269:242-244

6. Jin L, McLean PA, Neel BG, Wortis HH (2002) Sialic acid binding domains of $\mathrm{CD} 22$ are required for negative regulation of B cell receptor signaling. J Exp Med 195:1199-1205

7. Pillai S, Cariappa A, Pirnie SP (2009) Esterases and autoimmunity: the sialic acid acetylesterase pathway and the regulation of peripheral B cell tolerance. Trends Immunol 30:488-493

8. Nitschke L (2009) CD22 and Siglec-G: B-cell inhibitory receptors with distinct functions. Immunol Rev 230:128-143

9. Doucey MA, Scarpellino L, Zimmer J, Guillaume P, Luescher IF, Bron C, Held W (2004) Cis-association of Ly49A with MHC class I restricts natural killer cell inhibition. Nat Immunol 5:328-336

10. Karlhofer FM, Ribaudo RK, Yokoyama WM (1992) MHC class I alloantigen specificity of Ly-49+ IL-2 activated natural killer cells. Nature 358:66-70

11. Moretta A, Vitale M, Borrino C, Orengo A, Morelli L, Augugliaro R, Barbaresi M, Ciccone E, Moretta L (1993) P58 molecules as putative receptors for major histocompatibility complex (MHC) class I molecules in human natural killer (NK) cells. Antip58 antibodies reconstitute lysis of MHC class I-protected cells in NK clones displaying different specifities. J Exp Med 178: 597-604

12. Held W, Mariuzza R (2008) Cis interactions of immunoreceptors with MHC and non-MHC ligands. Nat Rev Immunol 8:269-278

13. Yin $Y$, Yamashita $Y$, Noda $H$, Okafuji $T$, Go MJ, Tanaka $H$ (2004) EphA receptor tyrosine kinases interact with co-expressed ephrin-A ligands in cis. Neurosci Res 48:285-296

14. Masuda A, Nakamura A, Maeda T, Sakamoto Y, Takai T (2007) Cis binding between inhibitory receptors and MHC class I can regulate mast cell activation. J Exp Med 204:907-920

15. Cordle J, Johnson S, Tay JZ, Roversi P, Wilkin MB, de Madrid BH, Shimizu H, Jensen S, Whiteman P, Jin B, Redfield C, Baron M, Lea SM, Handford PA (2008) A conserved face of the Jagged/ Serrate DSL domain is involved in Notch trans-activation and cisinhibition. Nat Struct Mol Biol 15:849-857

16. Cheung TC, Oborne LM, Steinberg MW, Macauley MG, Fukuyama S, Sanjo H, D’Souza C, Norris PS, Pfeffer K, Murphy KM, Kronenberg M, Spear PG, Ware CF (2009) T cell intrinsic heterodimeric complexes between HVEM and BTLA determine receptivity to the surrounding microenvironment. J Immunol 183:7286-7296

17. Haklai-Topper L, Mlechkovich G, Savariego D, Gokhman I, Yaron A (2010) Cis interaction between Semaphorin6A and Plexin-A4 modulates the repulsive response to Sema6A. EMBO J 29:2635-2645

18. Ljunggren HG, Kärre K (1990) In search of the 'missing self': MHC molecules and NK cell recognition. Immunol Today 11:237-244

19. Tormo J, Natarajan K, Margulies DH, Mariuzza RA (1999) Crystal structure of a lectin-like natural killer cell receptor bound to its MHC class I ligand. Nature 402:623-631

20. Matsumoto N, Mitsuki M, Tajima K, Yokoyama W (2001) The functional binding site for the C-type lectin-like natural killer cell receptor Ly49A spans three domains of its major histocompatibility complex class I ligand. J Exp Med 193:147-157

21. Wang J, Whitman MC, Natarajan K, Tormo J, Mariuzza RA, Margulies DH (2002) Binding of the natural killer cell inhibitory receptor Ly49A to its major histocompatibility complex class I ligand. Crucial contacts include both $\mathrm{H}-2 \mathrm{Dd}$ and beta 2-microglobulin. J Biol Chem 277:1433-1442

22. Back J, Chalifour A, Scarpellino L, Held W (2007) Stable masking by $\mathrm{H}-2 \mathrm{Dd}$ cis ligand limits Ly49A relocalization to the site of NK cell/target cell contact. Proc Natl Acad Sci USA 104:3978-3983
23. Andersson KE, Williams GS, Davis DM, Hoglund P (2007) Quantifying the reduction in accessibility of the inhibitory NK cell receptor Ly49A caused by binding MHC class I proteins in cis. Eur J Immunol 37:516-527

24. Hanke T, Takizawa H, McMahon CW, Busch DH, Pamer EG, Miller JD, Altman JD, Liu Y, Cado D, Lemonnier FA, Bjorkman PJ, Raulet DH (1999) Direct assessment of MHC class I binding by seven Ly49 inhibitory NK cell receptors. Immunity 11:67-77

25. Jonsson AH, Yang L, Kim S, Taffner SM, Yokoyama WM (2010) Effects of MHC class I alleles on licensing of Ly49A+ NK cells. J Immunol 184:3424-3432

26. Kim S, Poursine-Laurent J, Truscott SM, Lybarger L, Song YJ, Yang L, French AR, Sunwoo JB, Lemieux S, Hansen TH, Yokoyama WM (2005) Licensing of natural killer cells by host major histocompatibility complex class I molecules. Nature 436:709-713

27. Scarpellino L, Oeschger F, Guillaume P, Coudert JD, Levy F, Leclercq G, Held W (2007) Interactions of Ly49 family receptors with MHC class I ligands in trans and cis. J Immunol 178: $1277-1284$

28. Mori Y, Tsuji S, Inui M, Sakamoto Y, Endo S, Ito Y, Fujimura S, Koga T, Nakamura A, Takayanagi H, Itoi E, Takai T (2008) Inhibitory immunoglobulin-like receptors LILRB and PIR-B negatively regulate osteoclast development. J Immunol 181: $4742-4751$

29. Tai LH, Goulet ML, Belanger S, Toyama-Sorimachi N, FodilCornu N, Vidal SM, Troke AD, McVicar DW, Makrigiannis AP (2008) Positive regulation of plasmacytoid dendritic cell function via Ly49Q recognition of class I MHC. J Exp Med 205:3187-3199

30. Yoshizaki M, Tazawa A, Kasumi E, Sasawatari S, Itoh K, Dohi T, Sasazuki T, Inaba K, Makrigiannis AP, Toyama-Sorimachi N (2009) Spatiotemporal regulation of intracellular trafficking of Toll-like receptor 9 by an inhibitory receptor, Ly49Q. Blood 114:1518-1527

31. Sivori S, Falco M, Carlomagno S, Romeo E, Soldani C, Bensussan A, Viola A, Moretta L, Moretta A (2010) A novel KIR-associated fucntion: evidence rthat CpG DNA uptake and shuttling to early endosomes is mediated by KIR2DL2. Blood 116:1637-1647

32. Takai $\mathrm{T}$ (2005) A novel recognition system for MHC class I molecules constituted by PIR. Adv Immunol 88:161-192

33. Colonna M, Navarro F, Bellon T, Llano M, Garcia P, Samaridis J, Angman L, Cella M, Lopez-Botet M (1997) A common inhibitory receptor for major histocompatibility complex class I molecules on human lymphoid and myelomonocytic cells. J Exp Med 186:1809-1818

34. Huang J, Goedert JJ, Sundberg EJ, Cung TD, Burke PS, Martin MP, Preiss L, Lifson J, Lichterfeld M, Carrington M, Yu XG (2009) HLA-B*35-Px-mediated acceleration of HIV-1 infection by increased inhibitory immunoregulatory impulses. J Exp Med 206:2959-2966

35. Endo S, Sakamoto Y, Kobayashi E, Nakamura A, Takai T (2008) Regulation of cytotoxic $\mathrm{T}$ lymphocyte triggering by PIR-B on dendritic cells. Proc Natl Acad Sci USA 105:14515-14520

36. Olsson MY, Karre K, Sentman CL (1995) Altered phenotype and function of natural killer cells expressing the major histocompatibility complex receptor Ly-49 in mice transgenic for its ligand. Proc Natl Acad Sci USA 92:1649-1653

37. Fernandez NC, Treiner E, Vance RE, Jamieson AM, Lemieux S, Raulet DH (2005) A subset of natural killer cells achieve selftolerance without expressing inhibitory receptors specific for self MHC molecules. Blood 105:4416-4423

38. Anfossi N, Andre P, Guia S, Falk CS, Roetynck S, Stewart CA, Breso V, Frassati C, Reviron D, Middleton D, Romagne F, Ugolini S, Vivier E (2006) Human NK cell education by inhibitory receptors for MHC class I. Immunity 25:331-342 
39. Kim S, Sunwoo JB, Yang L, Choi T, Song YJ, French AR, Vlahiotis A, Piccirillo JF, Cella M, Colonna M, Mohanakumar T, Hsu KC, Dupont B, Yokoyama WM (2008) HLA alleles determine differences in human natural killer cell responsiveness and potency. Proc Natl Acad Sci USA 105:3053-3058

40. Ohlen C, Kling G, Höglund P, Hansson M, Scangos G, Bieberich C, Jay G, Kärre K (1989) Prevention of allogeneic bone marrow graft rejection by $\mathrm{H}-2$ transgene in donor mice. Science 246 : 666-668

41. Raulet DH, Vance RE (2006) Self-tolerance of natural killer cells. Nat Rev Immunol 6:520-531

42. Chalifour A, Scarpellino L, Back J, Brodin P, Devèvre E, Gros F, Lévy F, Leclercq G, Höglund P, Beermann F, Held W (2009) A role for cis interaction between the inhibitory Ly49A receptor and MHC class I for NK cell education. Immunity 30:337-347

43. Vyas YM, Maniar H, Lyddane CE, Sadelain M, Dupont B (2004) Ligand binding to inhibitory killer cell Ig-like receptors induce colocalization with Src homology domain 2-containing protein tyrosine phosphatase 1 and interruption of ongoing activation signals. J Immunol 173:1571-1578

44. Fourmentraux-Neves E, Jalil A, Da Rocha S, Pichon C, Chouaib S, Bismuth G, Caignard A (2008) Two opposite signaling outputs are driven by the KIR2DL1 receptor in human CD4+ T cells. Blood 112:2381-2389

45. Tripathy SK, Keyel PA, Yang L, Pingel JT, Cheng TP, Schneeberger A, Yokoyama WM (2008) Continuous engagement of a self-specific activation receptor induces NK cell tolerance. J Exp Med 205:1829-1841

46. Egea J, Klein R (2007) Bidirectional Eph-ephrin signaling during axon guidance. Trends Cell Biol 17:230-238

47. Sprinzak D, Lakhanpal A, Lebon L, Santat LA, Fontes ME, Anderson GA, Garcia-Ojalvo J, Elowitz MB (2010) Cis- interactions between Notch and Delta generate mutually exclusive signalling states. Nature 465:86-90

48. Montgomery RI, Warner MS, Lum BJ, Spear PG (1996) Herpes simplex virus-1 entry into cells mediated by a novel member of the TNF/NGF receptor family. Cell 87:427-436

49. Cosman D, Fanger N, Borges L, Kubin M, Chin W, Peterson L, Hsu ML (1997) A novel immunoglobulin superfamily receptor for cellular and viral MHC class I molecules. Immunity 7: 273-282

50. Back J, Malchiodi EL, Cho S, Scarpellino L, Schneider P, Kerzic MC, Mariuzza RA, Held W (2009) Distinct conformations of Ly49 natural killer cell receptors mediate MHC class I recognition in trans and cis. Immunity 31:598-608

51. Natarajan K, Dimasi N, Wang J, Mariuzza RA, Margulies DH (2002) Structure and function of natural killer cell receptors: multiple solutions to self, nonself discrimination. Annu Rev Immunol 20:853-885

52. Deng L, Mariuzza RA (2006) Structural basis for recognition of MHC and MHC-like ligands by natural killer cell receptors. Semin Immunol 18:159-166

53. Meijers R, Puettmann-Holgado R, Skiniotis G, Liu JH, Walz T, Wang JH, Schmucker D (2007) Structural basis of Dscam isoform specificity. Nature 449:487-491

54. Schwartz JC, Zhang X, Nathenson SG, Almo SC (2002) Structural mechanisms of costimulation. Nat Immunol 3:427-434

55. Deng L, Cho S, Malchiodi EL, Kerzic MC, Dam J, Mariuzza RA (2008) Molecular architecture of the MHC-binding site of Ly49 natural killer cell receptors. J Biol Chem 283:16840-16849

56. Boyington JC, Motyka SA, Schuck P, Brooks AG, Sun PD (2000) Crystal structure of an NK cell immunoglobulin-like receptor in complex with its class I MHC ligand. Nature 405:537-543 